Document downloaded from:

http://hdl.handle.net/10251/67783

This paper must be cited as:

Moreno, V.; Mora Almerich, J.; Muriel, M.; Capmany Francoy, J. (2015). Scalable HighOrder UWB Pulse Generation Employing an FBG-Based Photonic Superstructure. IEEE Photonics Technology Letters. 27(20):2146-2149. doi:10.1109/LPT.2015.2454952.

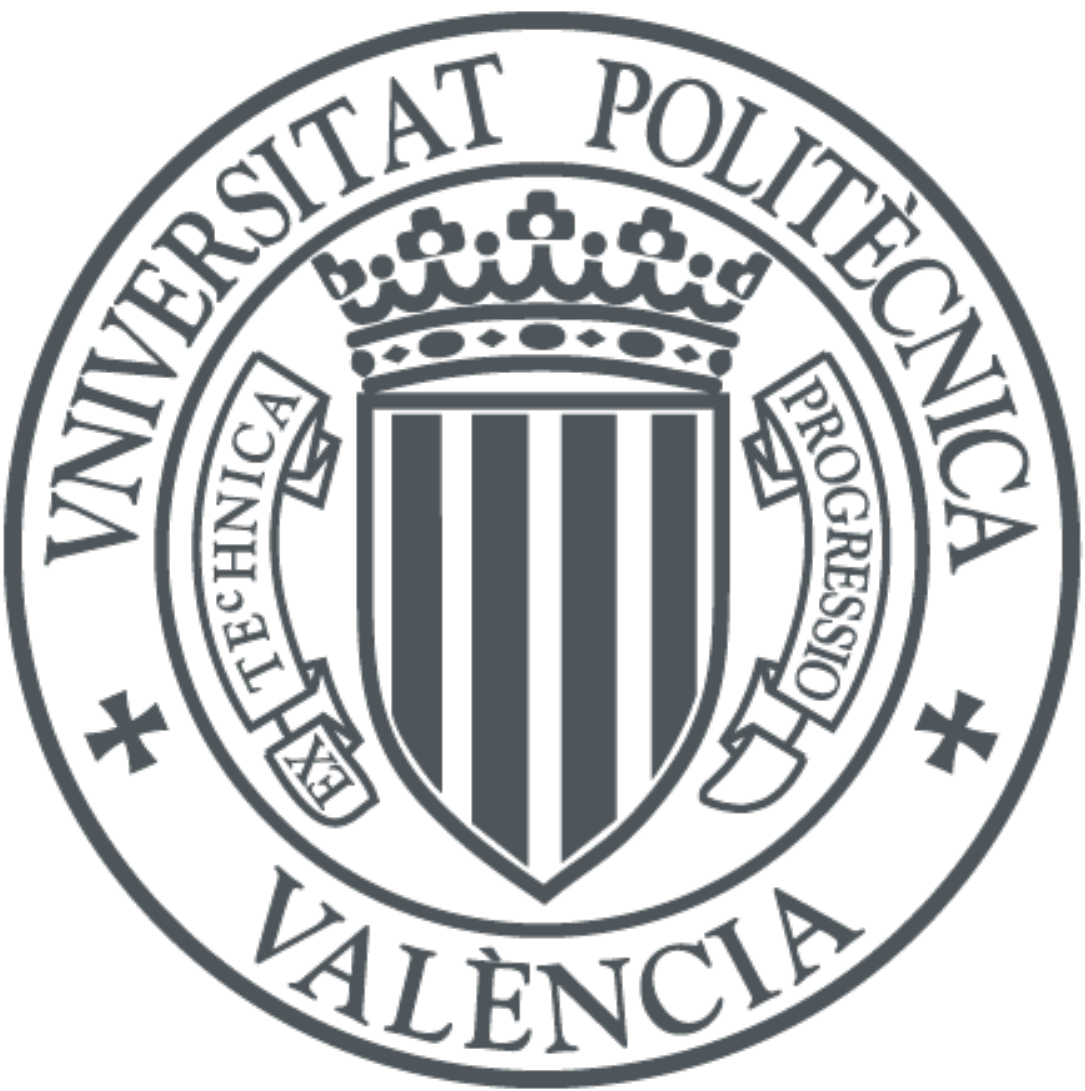

The final publication is available at

http://dx.doi.org/10.1109/LPT.2015.2454952

Copyright Institute of Electrical and Electronics Engineers (IEEE)

Additional Information 


\section{Scalable High-Order UWB Pulse Generation Employing an FBG-Based Photonic Superstructure}

Vanessa Moreno, Jose Mora, Miguel A. Muriel, and Jose Capmany

\begin{abstract}
In this letter, we propose and experimentally demonstrate a compact, flexible, and scalable ultrawideband (UWB) generator based on the merge of phase-to-intensity conversion and pulse shaping employing an fiber Bragg Grating-based superstructure. Our approach offers the capacity for generating high-order UWB pulses by means of the combination of various low-order derivatives. Moreover, the scheme permits the implementation of binary and multilevel modulation formats. Experimental measurements of the generated UWB pulses, in both time and frequency domain, are presented revealing efficiency and a proper fit in terms of Federal Communications Commission settled standards.
\end{abstract}

Index Terms-Fiber optics, microwave photonics, photonic superstructure.

\section{INTRODUCTION}

$\mathbf{U}$ LTRAWIDEBAND (UWB) technology exploitation has been present in a vast range of applications areas for some time due to its versatile applicability [1]. Its potentiality is related to transmiting high data rates, low power consumption, immunity to multipath fading, interference mitigation, low loss, carrier free and especially because of its spectrum cohabitation capacity with other narrowband technologies [2]. An UWB pulse must hold a fractional bandwidth larger than $20 \%$ or a $10 \mathrm{~dB}$ bandwidth of at least $500 \mathrm{MHz}$ in the frequency range from 3.1 to $10.6 \mathrm{GHz}$ associated to an EIRP of $-41.3 \mathrm{dBm} / \mathrm{MHz}$ [3]. This implies a tradeoff in terms of transmission distance and high speed communications. In order to overcome this coverage limitation, microwave photonics (MWP) has been introduced as a key partner of UWB system that permits to fully exploit the inherent advantages provided by operating directly in the optical domain such as light weight, small size, and immunity to electromagnetic interference with no need for extra electrical to optical conversion [4].

Since MWP is established as a reliable technology that provides valuable features such as flexibility and reconfigurability [5], it has constituted a solid base for different UWB signal generation and modulation approaches in the last years [6].

Initially, UWB monocycles and doublets have been considered standard waveforms for UWB applications. However, the generation of high-order pulses is found more interesting since they are capable of satisfying fully Federal Communications Commission (FCC) regulations [7]. Among the most relevant techniques related to obtain such highorder pulses, we can find microwave photonic filtering [8], nonlinearities exploitation by means of Semiconductor Optical Amplifiers (SOAs) [9] and PM-IM conversion based schemes [10]. At the same time, these approaches can be classified into those which require the insertion of one coefficient into the system for each Gaussian based pulse [8], [9] and those designs that consist on the merge of various low order UWB pulses, such as monocycles and doublets [10]-[12]. On one hand, the first ones based on Gaussian pulses do not comply with fully wavelength efficiency or rely on complex non-linear effects that disable any reconfiguration capability. On the other hand, the last ones are more competent when compared to the before mentioned schemes. However, the current architectures based on these last architectures present some deficiencies such as lack of flexibility, low reconfiguration and finally, limited integration feature.

The concept of combining phase modulation with optical filters has been approached previously [13], [14] but high order pulses generation has not been experimentally reported. In the case of [13], the PM-IM conversion takes place in a single FBG, constraining any possibility of high-order pulses creation. As for [14] this design reveals a complex receiver due to the presence of a balanced photodetector (BPD). Therefore, such bulky architecture diminishes the possibilities of an integrated optics approach. In this letter, a flexible photonic architecture to generate high order UWB pulses employing a Fiber Bragg Gratings (FBGs) based superstructure has been proposed and experimentally demonstrated. The principle of operation relies on the combination of a phase modulator with a customized FBG array for a simultaneous process of PM-IM conversion and pulse shaping. Section 2 describes the principle of operation considering each one of the conversion operation points and the impact of different FBG design parameters. In section 3, experimental results are discussed. These results show that scalability in this implementation is directly related to the number of optical sources and FBGs within the array employed. Apart from the generation of pulses with an efficient compliance of the FCC spectral mask, this 


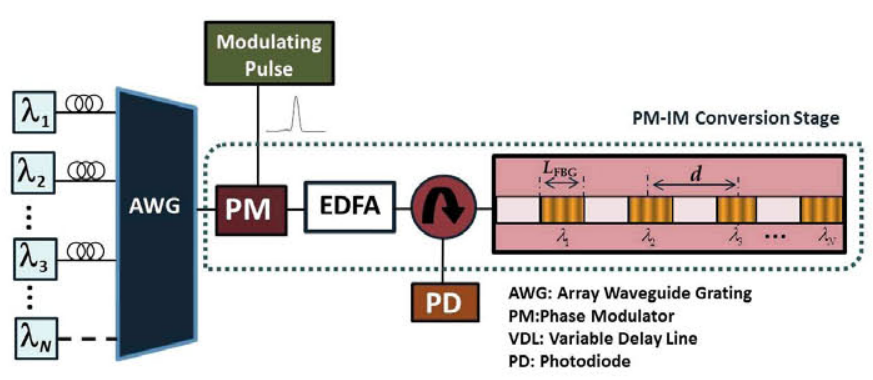

Fig. 1. General layout for proposal focused on high-order UWB pulses generation based on a FBG photonic superstructure.

optical UWB system holds the possibility of pulse codification, implementing different modulation formats such as Pulse Position Modulation (PPM), Bi-Phase Modulation (BPM) and Pulse Shape Modulation (PSM) which will be addressed at the end of this experimental section. Finally, conclusions are exposed in section 4 .

\section{PRINCIPLE OF OPERATION}

Figure 1 illustrates a scheme of the proposed high-order pulses generator with modulation capabilities by employing a FBG-based superstructure. In the layout, the optical signal from a set of "N" optical sources is launched to a phase modulator (PM) by means of a multiplexing stage, represented in this case by an Array waveguide grating (AWG). The PM is fed by an electrical Gaussian pulse sequence with a fixed pattern of one " 1 " and sixty three "0" (total 64 bits) at a $12.5 \mathrm{~Gb} / \mathrm{s}$ bit rate and a RF Drive Pulse Amplitude of $0.250 \mathrm{Vpp}$. An important parameter to consider in this stage prior to the phase modulation step, it's the necessity of polarization control mechanisms for the operational wavelengths involved, this matter is addressed through individual polarization controllers (PCs) for each one of the lasers sources. The PM device has an insertion loss of $3.5 \mathrm{~dB}$ and a 3dB-bandwidth of $20 \mathrm{GHz}$. Once modulated, the obtained pulse is amplified with an Erbium Doped Fiber Amplifier (EDFA) and introduced to the FBG superstructure through an optical circulator. The assemblage will perform the desired PM-IM conversion in function of the optical wavelength selected for the employed optical sources.

The mentioned FBG structure consists on an array constituted by $N$ FBGs designed with an apodized profile considering a precise separation " $d$ " and length " $l$ " in order to maximize the desired PM-IM conversion with an exact optical delay between adjacent optical taps for pulse shaping.

In this implementation, we expand the concept of PM-IM conversion with a single fiber Bragg grating (FBG) operating as a frequency discriminator [13] by introducing high order pulses generation and modulation capacities through the FBG superstructure. There is an intrinsic relation between the input wavelengths and the Bragg wavelengths of the FBGs that compose the customized photonic shaper. Experimentally, we have considered four (04) laser sources with a wavelength stability of tens of pm and a customized FBG superstructure comprised by 4 concatenated FBGs, hence $\mathrm{N}=4$. The power reflection spectrum of the complete array is depicted in Fig. 2. The design of a suitable FBG array is a significant matter since

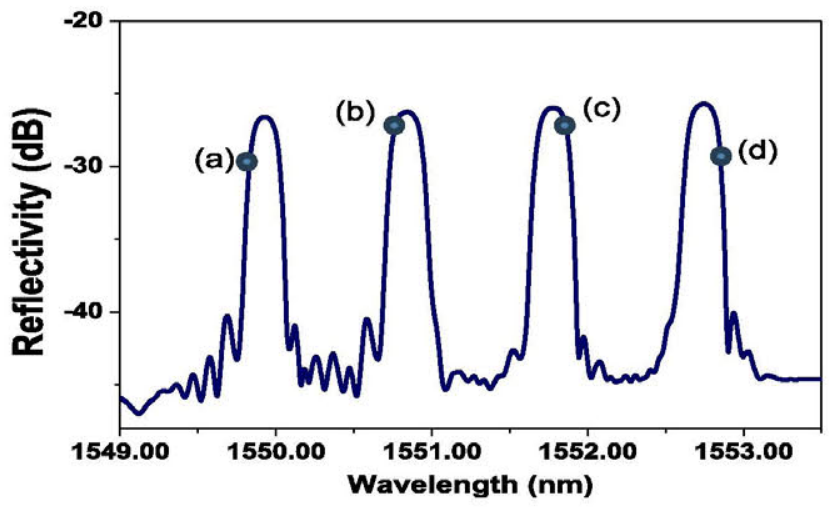

Fig. 2. Power reflection spectrum of the selected array. For instance, the four operations points (a), (b), (c) and (d) for each FBGs are indicated.

the quality of the reflected spectrum and the distance between each one will determine the quality of the high-order pulse to be produced. First of all, the separation " $d$ " represents a key value for this superstructure. This parameter was designed to be around $8 \mathrm{~mm}$ since the optical delay, " $\tau$ ", between optical taps holds a direct relationship with such factor. It was determined to be close to $77 \mathrm{ps}$, which corresponds to a Free Spectral Range $(F S R=1 / \tau)$ of approximately $12.9 \mathrm{GHz}$. Note that the FSR/2 around $6.45 \mathrm{GHz}$ matches with the central frequency of the expected UWB spectral band. The FBG design requires minimizing the crosstalk between channels, so a certain wavelength separation between these FBGs is needed. Spectra of each FBG are spectrally approximately equispaced as shown in Fig. 2.

The FBGs are centred at the wavelengths $\lambda_{\text {Bragg1 }}=$ $1549.808 \mathrm{~nm}, \lambda_{\text {Bragg } 2}=1550.712 \mathrm{~nm}, \lambda_{\text {Bragg } 3}=1551.656 \mathrm{~nm}$ and $\lambda_{\text {Bragg } 4}=1552.620 \mathrm{~nm}$. However, this wavelength separation is not enough to achieve reduced crosstalk. Furthermore, a specific apodized profile is required to reduce the secondary lobes of the FBG spectrum. The FBG inscription has been performed employing an Argon ion frequency-doubled laser at a wavelength of $244 \mathrm{~nm}$. The inscription procedure is based on the use of an uniform phase mask combined with a precise relative movement between the phase mask and the optical fiber [15]. This technique permits the apodization of the refractive index modulation along the optical fiber. A Kaiser apodization is employed in these FBGs in order to obtain the desired spectrum. The use of a boron co-doped optical fiber increases the photosensitivity achieving a reflectivity between $82 \%$ and $90 \%$. Finally, we evaluate the FBG shaper in function of the length. In this context, the length of the FBG is a flexible parameter. For this scheme, a $7 \mathrm{~mm}$ length $(l)$ is considered an optimal value of length for each FBG within the array in order to avoid overlapping between adjacent gratings in the fabrication process.

Flexibility of the system is demonstrated since each FBG permits to operate at different working points along its optical transfer function providing alternative pulse generation possibilities. A qualitative depiction of the PM-IM conversion process is illustrated in Fig. 3. In this scenario, by locating the optical carriers at the linear or quadrature slope of the FBG reflection spectrum, different waveforms are generated. 

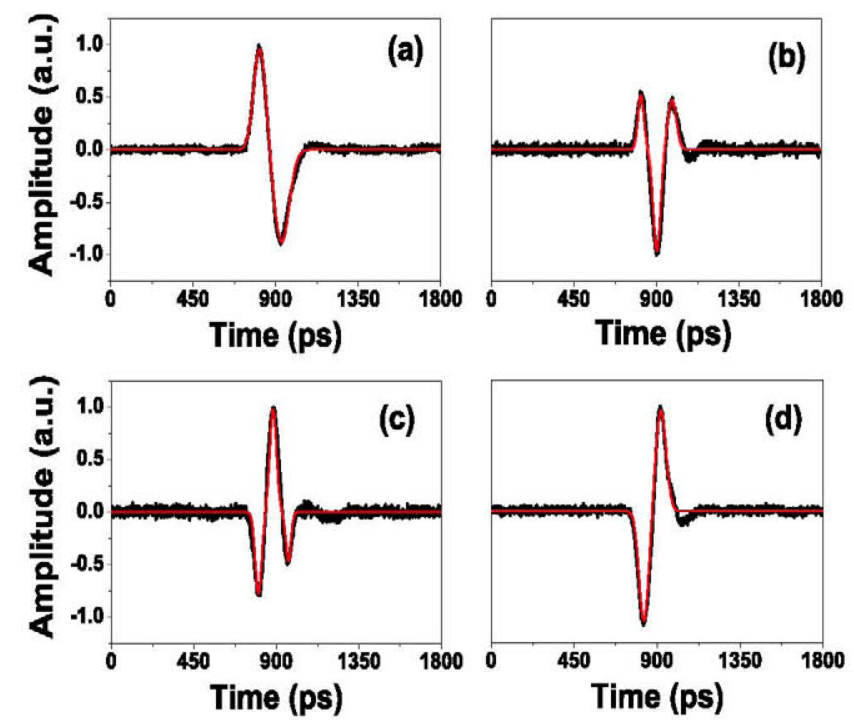

Fig. 3. Experimental measurements (black lines) and theoretical simulations (red lines) corresponding to the four obtained waveforms (a) monocycle, (b) doublet, (c) inverted doublet and (d) inverted monocycle.

For instance, FBG1 with operational point "a" achieves a monocycle, FBG2 provides a doublet by means of working point "b", FBG3 accomplishes a monocycle with opposite polarity to the one obtained with FBG1. Finally, FBG4 provides the corresponding inverse doublet for the waveform produced through FBG2. Figure 3 plots the experimental measurements and theoretical simulations corresponding to the four obtained waveforms and therefore the four operational points provided by the FBGs that comprised the structure. By changing the wavelength of each optical carrier inside the corresponding FBG, any kind of UWB pulse combination can be obtained.

\section{EXPERIMENTAL RESULTS}

To demonstrate and validate the capabilities for the generation and modulation of the proposed scheme, different signals were produced and analyzed as shown in Fig. 4 and Fig. 5. Firstly, we examined the lower-order waveforms by means of the different FBGs that compose the array with the aim of obtaining the exact wavelength values in which they are generated.

One of the main properties featured by the superstructure is its capacity of generating UWB high order pulses by combining several low-order ones. To achieve this waveform, the proposed set up only employed two lasers with an optical power value of $5.5 \mathrm{dBm}$ for each one. Activation of such optical sources is proportional to the number of lower order pulses to be reconfigured in the outcome signal. Wavelengths employed in this peculiar case were $1549.837 \mathrm{~nm}$ and $1550.683 \mathrm{~nm}$. These values are directly related with the base UWB waveforms to be employed and hence the PM-IM conversion working point. For instance, in this case a triplet was accomplished by means of the lineal sum of two inverted doublet pulses, corresponding to the " $\mathrm{C}$ " and " $\mathrm{B}$ " operation points. This UWB triplet depicts a fractional bandwidth of $99.8 \%$ with a spectral
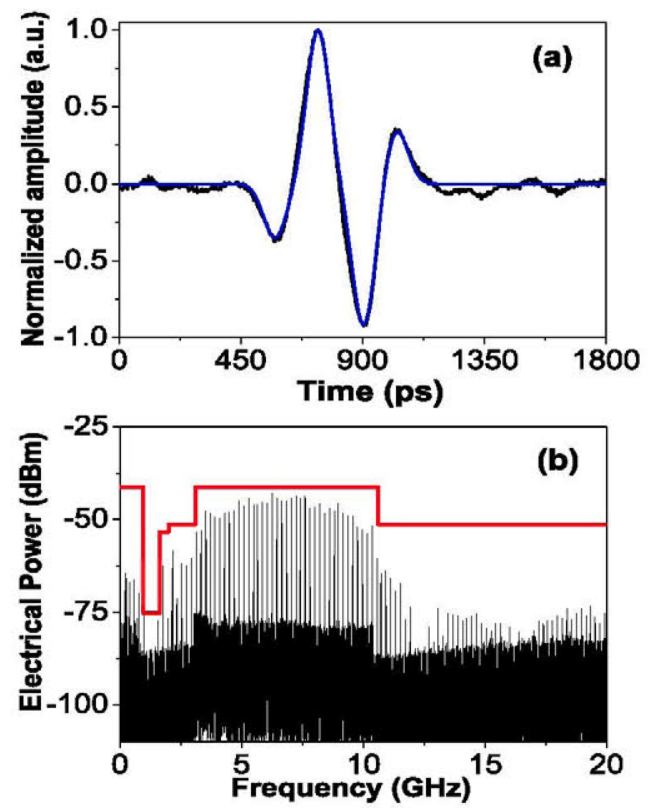

Fig. 4. (a) Experimental (black) and theoretical (blue) waveforms for the accomplished triplet pulse. (b) Corresponding experimental spectrum (black) of the mentioned triplet. FCC mask is plotted in red."
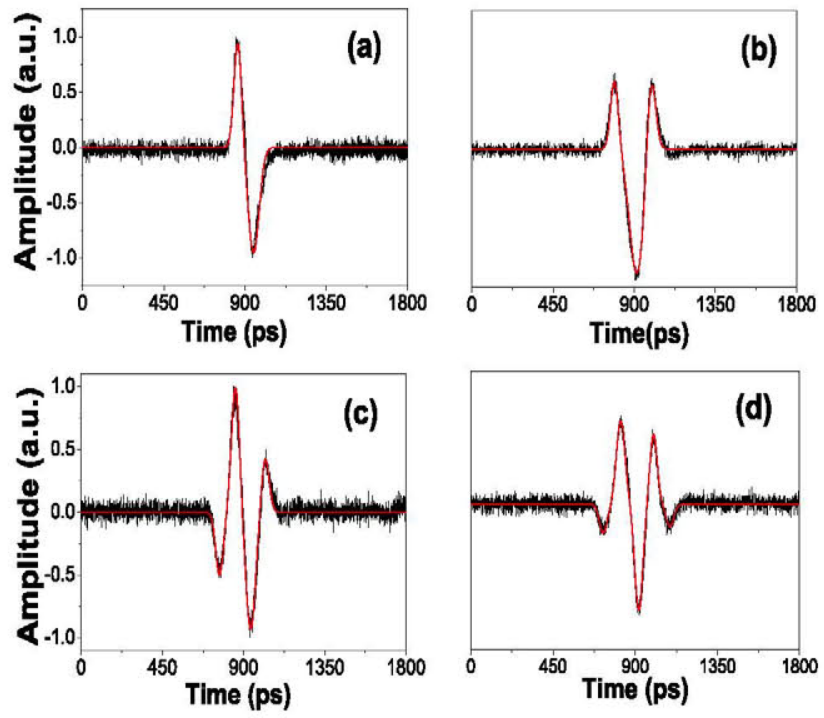

Fig. 5. (a) Four levels of pulse shape modulation: (a) monocycle, (b) doublet, (c) triplet and (d) a quadruplet. The pulses can be related to levels 00,01 , 01 and 11 .

efficiency of $65 \%$ in the $3.1-10.6 \mathrm{GHz}$ band. The temporal and frequency response of the obtained triplet pulse are displayed in Fig. 4(a) and Fig. 4(b), respectively.

In order to maximize the functionalities of the proposed system, along with high-order waveforms generation, implementation of diverse modulation formats such as Pulse Position Modulation (PPM), Bi-Phase Modulation (BPM) and pulse shape modulation (PSM) is also feasible. In the case of PPM, two, three or the whole four gratings are employed according to the number of time shifts aspired, separation between the original pulse and the delayed ones would locate at approximately 77 ps, 154 ps and 231 ps. In case of BPM, 
the system only requires to employ two gratings in order to accomplish this modulation format by wavelength tuning. We focus the experimental results in the performance of PSM because it shows on its own the full potentiality of our approach. Finally, a four level PSM scheme was also experimentally demonstrated. For this implementation, the system relies on the progressive activation of its optical sources (corresponding to wavelengths $\lambda_{1}, \lambda_{2}, \lambda_{3}$ and $\lambda_{4}$ ) and the capacity of generating lower and higher order UWB waveforms through the customized FBG superstructure Performance of this concept was carried out experimentally and its corresponding results are depicted in Fig. 5.

In this way, depending on the selected conversion point of the PM-IM cycle we can control the obtained pulse in the time domain. Therefore, by generating high order pulses it is possible to accomplish four different waveforms for an UWB PSM format. This reconfiguration process based on wavelength tuning can be explained as follows: in the case of Fig. 5(a), a monocycle was implemented through the use of a single laser which wavelength of $\lambda_{\mathrm{A} 1}=1549.721 \mathrm{~nm}$ was located at the resonance of FBG1. Then, this same optical source was set to $\lambda_{\mathrm{B} 1}=1549.771 \mathrm{~nm}$ in order to obtain the doublet depicted in Fig. 5(b). In the case of the third stage of modulation, the triplet was accomplished by means of the combination of operational points " $\mathrm{C}$ " and "B" of FBG1 and FBG2, this translates into activating simultaneously two lasers and setting the first one at $\lambda_{\mathrm{Cl}}=1549.837 \mathrm{~nm}$ and the second one at $\lambda_{\mathrm{B} 2}=1550.683 \mathrm{~nm}$, such waveform is depicted in Fig. 5(c). Finally, a quadruplet was created by means of the linear sum of three complementary doublets pulses, corresponding to the "B" and "C" operation points of three concatenated gratings at wavelengths $\lambda_{\mathrm{C} 1}=1549.837 \mathrm{~nm}, \lambda_{\mathrm{B} 2}=1550.683 \mathrm{~nm}$ and $\lambda_{\mathrm{C} 3}=1551.695 \mathrm{~nm}$. Just like with the triplet, all the optical lasers inputs involved were activated with a power value of $5.5 \mathrm{dBm}$. Fig. $5(\mathrm{~d})$ shows the system capability to generate a FCC mask compliant UWB quadruplet.

\section{CONCLUSIONS}

In this work, we have proposed and demonstrated a flexible high-order UWB pulse generation system by means of a photonic FBG superstructure. The key point is to take advantage of the benefits provided by the junction between a PM-IM technique with a pulse shaping structure. In our approach, the UWB pulses are produced through the combination of various low-order Gaussian derivatives and their complementary waveforms. The fundamental optical signals to generate the high-order pulses are monocycles and doublets which are accomplished through a PM-IM conversion process that takes place by means of a customized FBGs array. The design process of the FBG structure implies the proper selection of key parameters such as the separation, length and a specific apodized profile. We have experimentally proved the feasibility of this concept by implementing an UWB triplet signal which holds a spectrum compliant with FCC requirements. When comparing the doublet employed as the base lower order pulse and the generated UWB triplet, we observe an improvement in terms of spectral efficiency from $23 \%$ to $65 \%$ as expected. Parallel to this, the system portraits an interesting flexibility for adaptation to binary and multilevel modulation formats such as BPM, PPM and PSM.

\section{REFERENCES}

[1] S. Roy, J. R. Foerster, V. S. Somayazulu, and D. G. Leeper, "Ultrawideband radio design: The promise of high-speed, short-range wireless connectivity," Proc. IEEE, vol. 92, no. 2, pp. 295-311, Feb. 2004.

[2] D. Porcino and W. Hirt, "Ultra-wideband radio technology: Potential and challenges ahead," IEEE Commun. Mag., vol. 41, no. 7, pp. 66-74, Jul. 2003.

[3] J. Yao, "Photonics for ultrawideband communications," IEEE Microw. Mag., vol. 10, no. 4, pp. 82-95, Jun. 2009.

[4] S. Pan and J. Yao, "UWB-over-fiber communications: Modulation and transmission," J. Lightw. Technol., vol. 28, no. 16, pp. 2445-2455, Aug. 15, 2010.

[5] J. Capmany and D. Novak, "Microwave photonics combines two worlds," Nature Photon., vol. 1, no. 6, pp. 319-330, 2007.

[6] J. Yao, F. Zeng, and Q. Wang, "Photonic generation of ultrawideband signals," J. Lightw. Technol., vol. 25, no. 11, pp. 3219-3235, Nov. 2007.

[7] H. Sheng, P. Orlik, A. M. Haimovich, L. J. Cimini, and J. Zhang, "On the spectral and power requirements for ultra-wideband transmission," in Proc. IEEE Int. Conf. Commun. (ICC), May 2003, pp. 738-742.

[8] M. Bolea, J. Mora, B. Ortega, and J. Capmany, "Optical UWB pulse generator using an $N$ tap microwave photonic filter and phase inversion adaptable to different pulse modulation formats," Opt. Exp., vol. 17, no. 7, pp. 5023-5032, 2009.

[9] H. Feng, S. Xiao, L. Yi, and W. Hu, "Photonic generation of reconfigurable orders ultrawideband signals by using cascaded RSOAs," IEEE Photon. Technol. Lett., vol. 26, no. 9, pp. 908-910, May 1, 2014.

[10] V. Moreno, M. Rius, J. Mora, M. A. Muriel, and J. Capmany, "Scalable UWB photonic generator based on the combination of doublet pulses," Opt. Exp., vol. 22, no. 13, pp. 15346-15351, Jun. 2014.

[11] E. Zhou, X. Xu, K.-S. Lui, and K. K.-Y. Wong, "A power-efficient ultrawideband pulse generator based on multiple PM-IM conversions," IEEE Photon. Technol. Lett., vol. 22, no. 14, pp. 1063-1065, Jul. 15, 2010.

[12] S. T. Abraha, C. M. Okonkwo, E. Tangdiongga, and A. M. Koonen, "Power-efficient impulse radio ultrawideband pulse generator based on the linear sum of modified doublet pulses," Opt. Lett., vol. 36, no. 12, pp. 2363-2365, 2011

[13] F. Zeng and J. Yao, "Ultrawideband Impulse radio signal generation using a high-speed electrooptic phase modulator and a fiber-Bragggrating-based frequency discriminator," IEEE Photon. Technol. Lett., vol. 18, no. 19, pp. 2062-2064, Oct. 1, 2006.

[14] M. Abtahi, M. Mirshafiei, J. Magné, and L. A. Rusch, "Ultra-wideband waveform generator based on optical pulse-shaping and FBG tuning," IEEE Photon. Technol. Lett., vol. 20, no. 2, pp. 135-137, Jan. 15, 2008.

[15] M. J. Cole, W. H. Loh, R. I. Laming, M. N. Zervas, and S. Barcelos, "Moving fibre/phase mask-scanning beam technique for enhanced flexibility in producing fibre gratings with uniform phase mask," Electron. Lett., vol. 31, no. 17, pp. 1488-1490, Aug. 1995. 